\section{Acoustic estimates of Antarctic krill}

SIR-Everson et al.' discussed the implications of new measurements of target strength for estimating the abundance of krill in the Southern Occan. Their conclusions were first, that previously used equations $s^{2}$ relating target strength to physical size of these animals were greatly in error and, second, that the use of these equations has resulted in gross underestimates of krill abundance in the Southern Ocean. As krill provides the basis of a large fishery and is the main component of the diet of many marine predators, accurate estimates are essential for management of this resource. We have collected data covering a broad size range of crustacean zooplankton and micronekton, and verify and elaborate the findings of Everson et al. We present new target-strength-by-size relationships over the full size range of krill at the acoustical frequencies commonly used in field studies ${ }^{3}$

We collected data at $420 \mathrm{kHz}$ relating target strength to $\log k a$, where $k$ is the acoustical wavenumber and $a$ is the animal's equivalent spherical radius $(a$ in the figure). Although both theoretical and empirical studies indicate that the relationship between these two variables is nonlinear, a linear regression model relat-

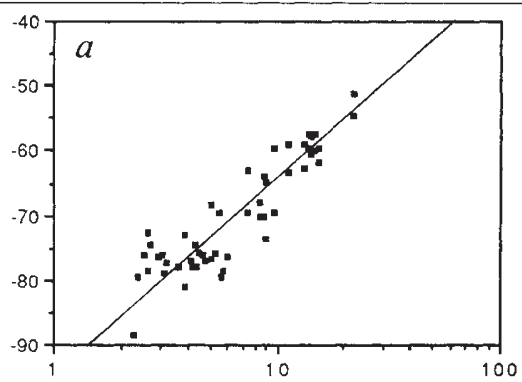

ka
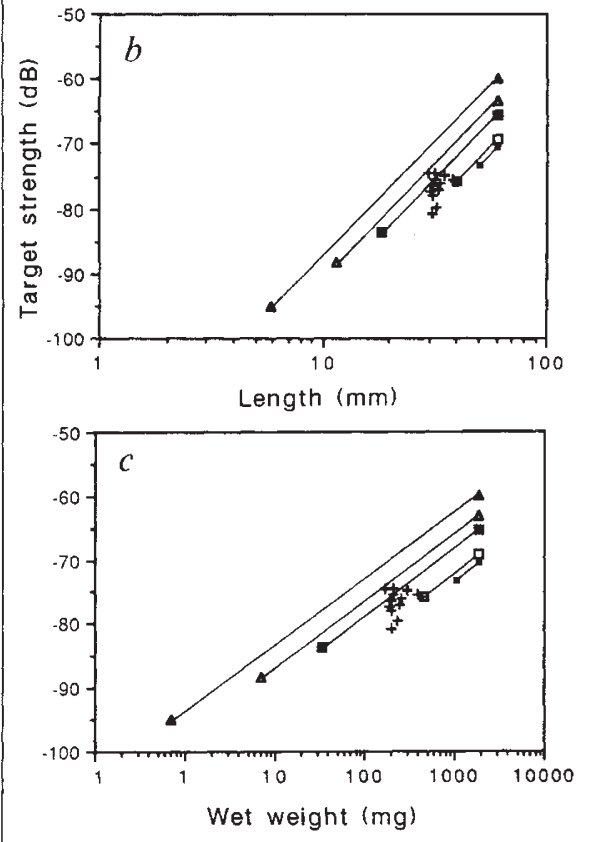

ing target strength to $\log k a$ cxplains a high proportion of the observed variance $\left(r^{2}=\right.$ 0.81 ) in the geometric scattering region where values of $k a$ exceed 1 (ref. 4). Some of the variance unexplained by the linearregression model can be associated with certain modal oscillations predicted by theoretical scattering models ${ }^{\dagger}$. Until these theoretical models are better developed, the linear model is of more practical use for predicting target-strength-by-size relationships. One attractive feature of the target-strength-by-log $k a$ relationship is that it can be manipulated, using a theoretically derived $10 \log k$ conversion factor ${ }^{\dagger}$, to predict how the target strength varies with animal size at a given acoustical frequency ( $b, c$ in the figure).

We note two points arising from the empirically derived relationships presented in the figure. First, the forms of the relationships imply that crustaccan zooplankton and micronekton scatter sound as a function of their volume rather than their cross-sectional area. This result, although consistent with most previous studies of sound scattering by crustacean zooplankton and micronekton ${ }^{+}$, is in conflict with the equations recommended for krill by the first international BIOMASS experiment (FIBEX) acoustic working

a, Target-strength (TS)-by-log ka relationship, with a linear regression model (TS $=-94.93+30.84$ $\log k a, n=52, r^{2}=0 ; 81$ ) fitted to data from acoustical experiments conducted with a broad size range of living crustacean zooplankton and micronekton". As frequency was held constant at $420 \mathrm{kHz}$ during these experiments, the regression slope of 30.84 applies to variations in $a$, not in $k$. Acousticai backscattering measurements were made on individual animals using the dual-beam method for TS estimation (see ref. 4 for experimental details). $b$, TS-by-log length relationships predicted at five acoustical frequencies commonly used in field studies. All relationships are truncated at a minimum length corresponding to values of $k a=1$ and a maximum length of $60 \mathrm{~mm}$. Data from ref. 1 collected at $120 \mathrm{kHz}$ are included, those at $38 \mathrm{kHz}$ are excluded. $c$. TS-by-log wet weight relationships predicted at five acoustical frequencies commonly used in field studies. All relationships are truncated at a minimum wet weight corresponding to values of $k a=1$ and a maximum wet weight of $1.900 \mathrm{mg}$. Data from ref. 1 collected at $120 \mathrm{kHz}$ are included, those at $38 \mathrm{kHz}$ are excluded. As the regression relationship reported in $a$ is derived from data collected at a single frequency, $420 \mathrm{kHz}$, a $10 \log k$ correction factor was applied to the relationship to predict TS values at other frequencies. This correction factor $(10 \mathrm{log}$ $k_{1} / k_{426}$, where $k_{t}$ is the wavenumber at the frequency of interest and $k$ is the wavenumber at $420 \mathrm{kHz}$, was derived from a linearized version of the straight finite cylinder scattering model described in ref. 4 . Values of equivalent spherical radius were converted directly to values of wet weight by the relationship: $W W=4.188 \times a^{3}$. Values of wet weight were then converted to values of length using the published relationship: $\log W W=-2.75$ $+3.39 \log L$ (ref. 5). b.c. From left to right, lines are at $420,200,120,50$ and $38 \mathrm{kHz}$. Crosses, data from ref. 1 at $120 \mathrm{kHz}$. group ${ }^{-}$Second, predicted target-strength values for various sizes of crustacean zooplankton and micronekton are considcrably lower than those predicted by the FIBEX equations. This finding verifies the general conclusions of Everson et al., despite some minor differences in the results of our two studies: Everson et al. estimated a slightly lower mean targetstrength value at $120 \mathrm{kHz}$ than we do, and they included results in the $30-40-\mathrm{mm}$ size range at $38 \mathrm{kHz}$.

The discrepancies at $120 \mathrm{kHz}$ can be partially accounted for by differences in experimental methods and/or failure to control for orientation effects in both studies. Also, while the animals in the two studies are anatomically similar, they are of different species, making direct comparison somewhat speculative. The differences at $38 \mathrm{kHz}$ are more fundamental. At this frequency, krill smaller than about 50 $\mathrm{mm}$ fall in the Rayleigh scattering region of the target-strength-by-log $k a$ relationship, in which target strength decreases precipitously with decreasing animal size. This results in a reduced ability to detect smaller animals as their echo levels fall below the inherent noise of the sonar system. For this reason, we recommend that only acoustical frequencies equal to or exceeding $120 \mathrm{kHz}$ be used for surveying the abundance of post-larval krill.

Thus, acoustical methods are well suited to the task of estimating krill abundance. but their use requires calibrated sonar systems and reliable target-strength-bysize relationships over the full size range of krill and at each of the acoustical frequencies being used. Although improvements can be made, the relationships presented here are the most reliable available and should enable others to interpret new acoustical survey data and to reanalyse older data sets more accurately.

ChARLES H. GREENE

Ocean Resources and Ecosystems

Program

Cornell University, Ithaca,

New York 14853, USA

TIMOTHY K. STANTON

Applied Ocean Physics and Engineering Department,

Biology Department,

Peter H. Wiebe

Woods Hole Oceanographic Institution. Woods Hole. Massachusetts 02543 USA

Department of Oceanography

SAM MCClatchiE

Dalhousie University, Halifax,

Nova Scotia, Canada B3H 4J1

Everson I. Watkins, J. Bone, D.G. \& Foote, K.G. Nature 345, 338-340 (1990)

2. Post-Fibex Acoustic Workshop. BIOMASS Report Ser. No. 40 (1986).

3. Miller, D.G.M. \& Hampton, 1. Biology and Ecology of the Antarctic Krill BIOMASS Scientific Ser. No. 9 (1988)

4. Wiebe, P.H., Greene, C.H., Stanton, T.K. \& Burczynski, J. J. acoust. Soc. Am. 88, 2346-2360 (1990).

5. Mac'ntosh. N.A. Discov. Rep. 36. 95-156 (1973). 